\title{
VIRTUAL APPLIANCE CREATION AND OPTIMIZATION IN CLOUD
}

\author{
Mohan Raj. $\mathbf{B}^{1}$, Radha. $\mathbf{N}^{2}$, Poongodi. $\mathbf{P}^{3}$ \\ ${ }^{1} P G$ scholar, Mahendra Engineering College \\ ${ }^{2}$ Ph.D scholar, Mahendra Engineering College \\ ${ }^{3}$ Prof HOD/ECE, PPG institute of technology
}

\begin{abstract}
The large scale IaaS systems could store virtual appliances in several repositories. The deployment time could heavily vary depending on the connection properties of the repository storing the appliance. A virtual appliance is a virtual machine image designed to run on a virtualization platform i.e. Virtual Box, Xen, VMware Workstation. Virtual appliance delivery requires the modification of the underlying IaaS systems. IaaS is the virtual delivery of computing resources in the form of hardware, networking, and storage services This concept will reduce the variance in deployment time by introducing the concept of online active repositories and appliance optimization. To provide efficient delivery time in IaaS and to increase the efficiency of IaaS (Infra structure as a service) system To calculate the delivery time when deployed it in the virtualized platform Combining the both online and manual repositories for calculating the delivery time To construct the appliance in online using various online repositories The constructed appliance is deployed in the virtualized platform (using virtual box). And the appliance is optimized for increasing the efficiency and decreasing the delivery time. The delivery time of the online appliance is compared with the appliance which has been created manually.
\end{abstract}

Keywords- Cloud Computing, Virtualization, Virtual Appliance.

\section{INTRODUCTION}

Cloud computing a relatively recent term, defines the paths ahead in Computer Science world. Being built on decades of research it utilizes all recent achievements in Virtualization, Distributed computing, Utility computing, and Networking. It implies a service oriented architecture through offering software and platforms as services (i.e SAAS, PAAS) reduced information technology overhead for the end-user, great flexibility, reduced total cost of ownership, on demand services and many other things.

The Cloud is a metaphor for the Internet, based on how it is depicted in computer network diagrams, and is an abstraction for the complex infrastructure it conceals. Cloud Computing becomes widespread particularly as these systems are "always-on always-available. "The Cloud is a Virtualization of resources that maintains and manages itself, delivering massively scalable enterprise IT as a Service across Internet.

\section{VIRTUALIZATION}

Virtualization is the creation of a virtual (rather than actual) version of something, such as an operating system, a server, a storage device or network resources. Virtualization is a technology that combines or divides computing resources to present one or many operating environments. It uses methodologies like hardware and software partitioning or aggregation, partial or complete machine simulation, emulation, time-sharing etc. Offers a wide variety of tangible benefits to those wanting to consolidate their computing resources and at the same time save money. E.g. VMware, Virtual PC, Virtual Box etc

\section{VIRTUAL APPLIANCE}

It is a virtual machine image, designed to run on a virtualization platform. Virtual appliances are a subset of a broader class of software appliances. Installation of a software appliance on a virtual machine creates a virtual appliance. Like software appliances, virtual appliances are intended to eliminate the installation, configuration and maintenance costs associated with running complex stacks of software.

Because virtual appliances are preconfigured, they help organizations reduce the time and expense associated with application deployment including the patching and ongoing management of the software. Delivering software as a virtual appliance has numerous business and technical benefits that can result in higher conversion rates, shorter sales cycles and increased renewals. In the virtual-appliance model, you can enable greater usability out of the box by optimizing the application for one of several VMware supported OS. KVM is a full virtualization solution for $\mathrm{x} 86$ processors supporting hardware virtualization. It consists of two main components VM Guests, Virtual storage and networks can be managed with libvirt based and QEMU tools. libvirt is a library that provides an API to manage VM Guests based on different virtualization solutions, among them KVM and Xen. It offers a graphical user interface as well as a command line program. The QEMU tools are KVM/QEMU specific and are only available for the command line. 


\section{PROJECT DESCRIPTION}

\subsection{Existing System}

The concept of virtualization describes the design and prototype of an extendable management framework, virtualization integrator (VSI), which enables an appliance builder to easily fulfill the key features of virtual appliances and coordinate management function across components located in multiple virtual appliances. And the main drawback of this paper is that virtual appliance is reduces the efficiency of the cloud data center. And therefore it will underlay some changes in the data center. So that it directly does not involve in the process of creation of virtual appliance, and in the next technique it introduces the concept of how to manage the virtual appliances and what are the steps for managing the virtual appliances.

\subsection{Proposed System}

In the proposed system we have explained the process of how the time of the virtual appliance is used and explained with the automated virtual appliance creation system, the deployment time reduction capabilities of the proposed techniques were measured with several services provided in virtual appliances on three cloud infrastructures.

The appliance creation capabilities of the VAS are compared to the already available virtual appliances offered by the various online appliance repositories. As a result these techniques alleviated one of the major obstacles before virtual appliance based deployment system.

\section{MODULES}

\subsection{Java Application Creation}

This module describes about creation of a java application. In this paper we have created an any java application for the IP hacker. This application contains a hit list with the attributes of Ip address, Time, Date in which the person access the system. If any ip address request or access data more than particular count it will restrict that ip address and it will say unauthorized access and intimate to admin. So we blocked ip address easily and efficiently.

\subsection{Appliance Creation}

The goal of this online appliance creation is to create the virtual appliance (software image) for a particular os or an application. The created virtual appliance will be deployed in the virtualized platform for calculating the delivery time. Virtual box is a tool for importing the appliance in any of the operating systems like (windows, Linux etc.,).The created online appliance is imported in the virtual box. The installation of the appliance begins in the cloud environment.

\subsection{Optimization of Virtual Appliance}

As from the first step of the optimization algorithm, it starts with the identification of the deployment time reduction options for services encapsulated in virtual appliances. The deployment time is defined as the time between the deployment decisions was made and the service was activated on the selected host. These tasks include the installation, the configuration and finally the activation. With these tasks, the installation is the most time consuming thus it is subdivided into several subtasks.

This approach modifies the virtual appliance in a way that it is capable of serving its target functionality, with a smaller size. A virtual appliance consist of a disk image, if there is a memory snapshot. Appliance optimization is done by the VA optimization facility that is a subsystem of the VAS service therefore; first, we defined the faults that can be injected in order to achieve size reduction. Having a virtualized environment enables the simulation of both software and hardware level faults.

Validation algorithms are used to evaluate these virtual appliance, to check whether it provides the target functionality. Software development may involve unit and integration tests optimization technique, which is represented in the updates and reusing the previous optimization results. The facility takes snapshots of the optimization process that create intermediate appliances.

\subsubsection{Appliance Contents Removal}

This section describes the basic algorithm of the optimization procedure is split into three distinct tasks. These three tasks are outlined in the next sub-sections.

\subsubsection{Selection}

Items are the smallest entities handled by the selection and removal algorithms. The subtask of the selection is weighting that prioritizes the different items. Weight functions assign weight values for each item of the virtual appliance under optimization. The higher the weight value the more undesired the actual item is in the virtual appliance. The optimization facility decides on the use of the various weight functions based on the time and cost constraints specified in the optimization target criterion.

\subsubsection{Removal and Validation}

The removal action sorts the items according to their weights and it removes the item with the highest weight from the virtual appliance. For the removal operation, the optimization facility has to understand the contents of the appliance to be able to remove the selected item. After removal, validation instantiates the reduced appliance in a virtual machine (vm) and all the developer-supplied validation algorithms validator 
are executed on the service it offers. If any of these algorithms fail, then the validation procedure is non-successful valid false.

Appliance contains several packages in that optimize the replicated data. Select weight in KB and check replicated data if anything found means remove that replicated data and provide call back function to original data or package. By doing we have reduced the size, while deploying there by this also reduce the time.

\subsection{Deploying into Cloud}

Finally we have deployed MMVA into a cloud system and compare the results with following key factors: the number of files, size and deployment time between application, virtual appliance and MMVA.

\section{RESULTS}

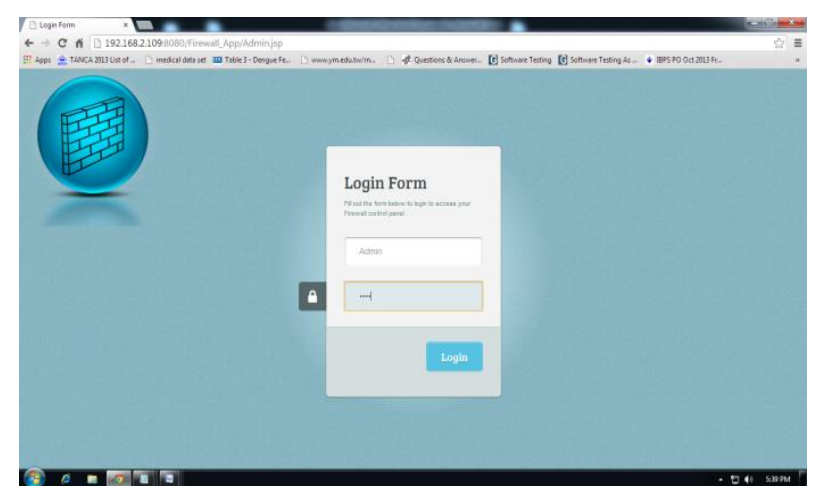

Fig 1 Admin Login Page

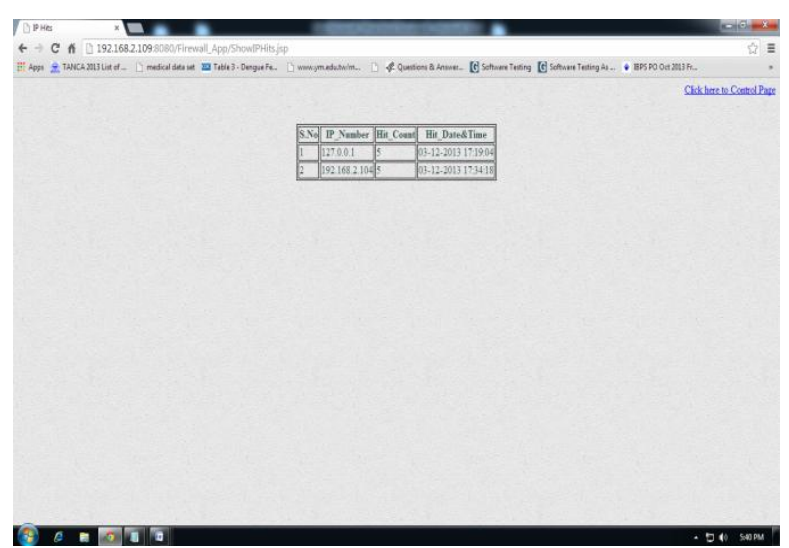

Fig 2 Hit List with IP Address

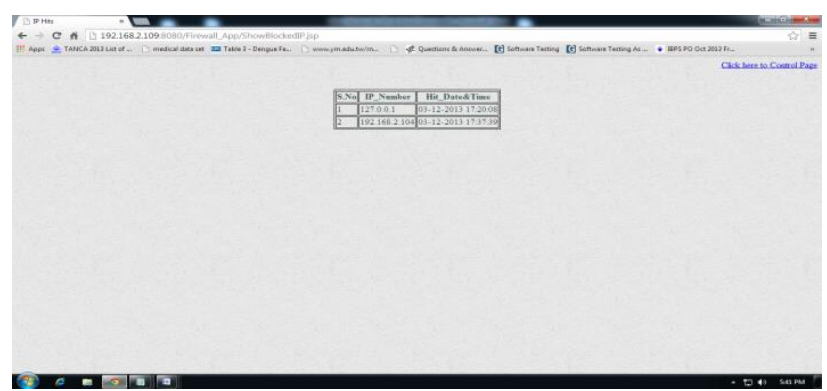

Fig 3 Blocked IP Addresses

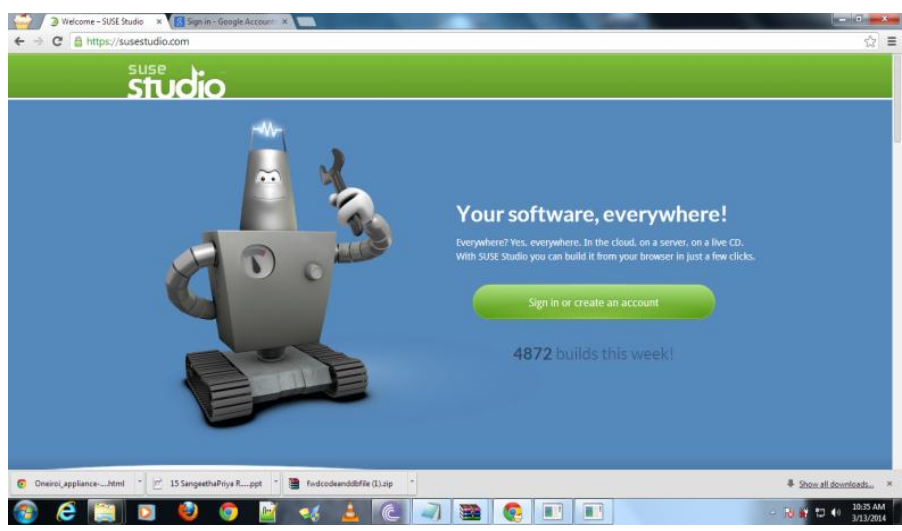

Fig 4 Convert appliance into iso file format

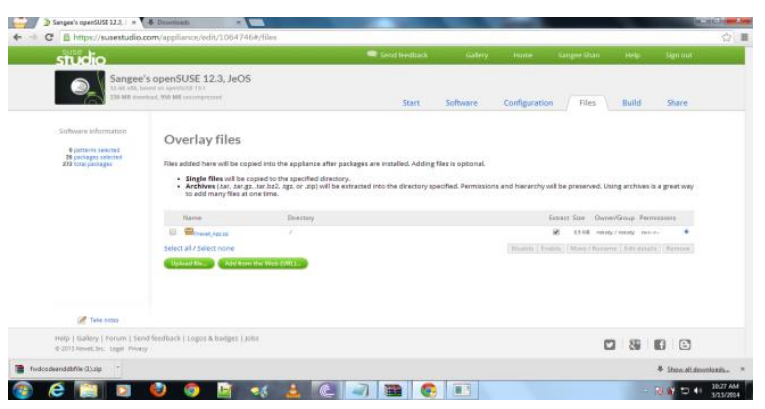

Fig 5 Uploading Application

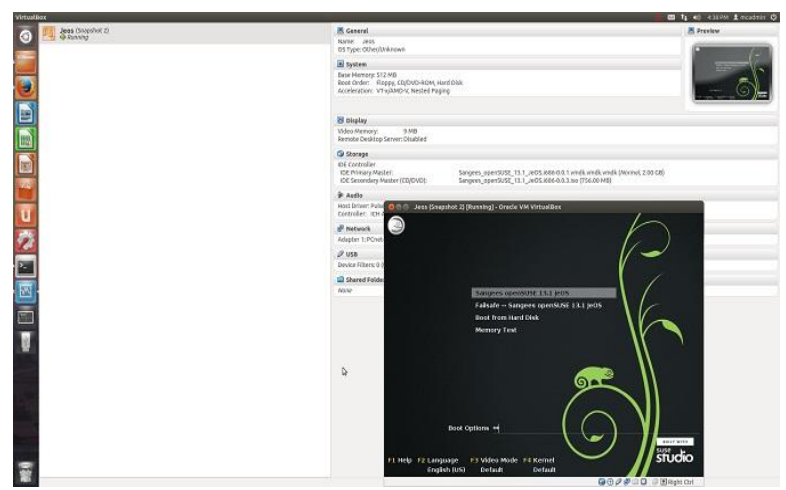

Fig 6 Importing appliance using virtual box 


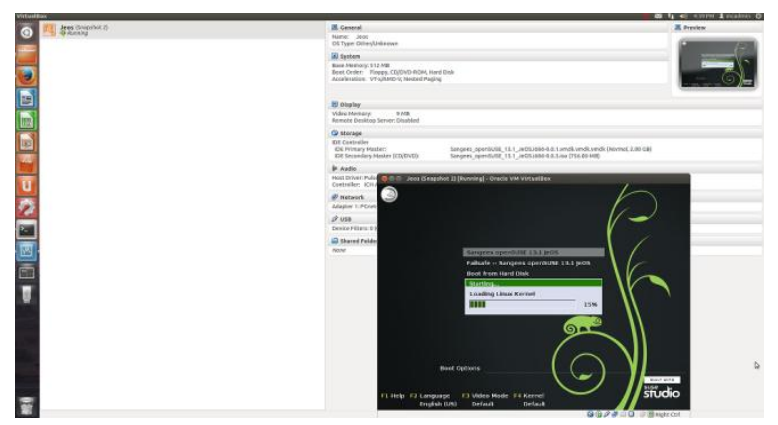

Fig 7 Booting of appliance

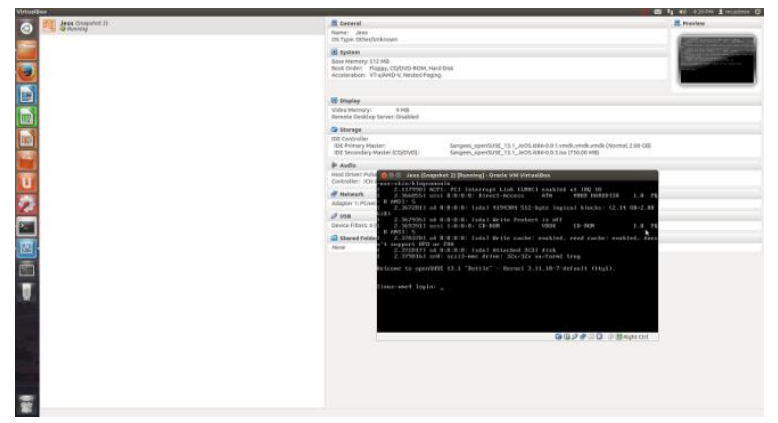

Fig 8 Appliance loaded

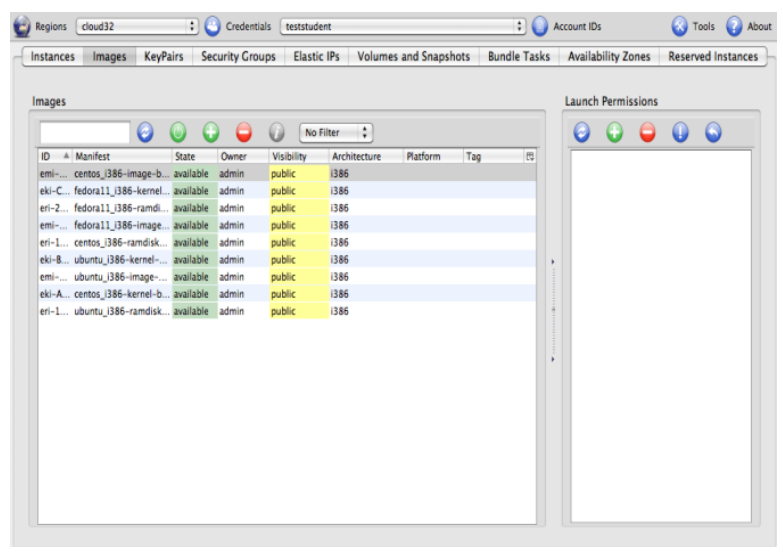

Fig 9 Cloud Deployment

\section{CONCLUSIONS}

Highly dynamic service environments introduce new demands on service deployment systems because they might schedule service calls on yet-to-be deployed service instances. Therefore, in highly dynamic service environments appliance based service deployment systems are only usable if the deployment time of the various appliances can be reduced.

In the current practice the virtual appliance creation task is usually performed manually that hinders dynamic service deployment and makes impossible to create dynamic adaptive systems. Also our contribution provided a "parallel algorithm for virtual appliance size optimization", this not only reduces the deployment time by optimizing the size of the virtual appliances, also provides a technique that minimizes the optimization time and allows the early release of the optimal appliances. And the future enhancement is increasing the efficiency and decreasing the delivery time using MMVA (minimal manageable virtual appliance).

\section{REFERENCES}

[1]. L. M. Vaquero, L. Rodero-Merino, J. Caceres et al., "A break in the clouds: towards a cloud definition," SIGCOMM Computer Communication Review, vol. 39, pp. 50-55, December 2008.

[2]. M. Armbrust, A. Fox, R. Griffith et al., "Above the clouds: A Berkeley view of cloud computing," University of California at Berkley, Tech. Rep. UCB/EECS-2009-28, February 2009.

[3]. N. Susanta and C. Tzi-Cker, "A survey on virtualization technologies," ECSL-TR-179, Stony Brook University, Tech. Rep., February 2005. [Online]. Available:

http://www.ecsl.cs.sunysb.edu/tr/TR179.pdf

[4]. Amazon Web Services LLC, "Amazon elastic compute cloud," http://aws.amazon.com/ec2/, 2012.

[5]. K. Keahey, I. Foster, T. Freeman, X. Zhang, and D. Galron, "Virtual workspaces in the grid," ANL/MCS-P12310205, 2005.

[6]. C. Sapuntzakis, D. Brumley, R. Chandra et al., "Virtual appliances for deploying and maintaining software," in LISA '03: Proceedings of the 17th USENIX conference on System administration. Berkeley,CA, USA: USENIX Association, 2003, pp. 181-194.

[7]. T. Zhanga, Z. Dua, Y. Chenb, X. Jic, and X. Wang, "Typical virtual appliances: An optimized mechanism for virtual appliances provisioning and management," The Journal of Systems and Software,vol. 84, pp. 377-387, 2011.

[8]. H. Nishimura, N. Maruyama, and S. Matsuoka, "Virtual clusters on the fly - fast, scalable, and flexible installation," in Proceedings of the Seventh IEEE International Symposium on Cluster Computing and the Grid, ser. CCGRID ' 07. Washington, DC, USA: IEEE Computer Society, 2007, pp. 549-556.

[9]. Vizioncore Inc., "voptimizer, optimization of virtual machine size and performance," 2008.

[10]. R. Bradshaw, N. Desai, T. Freeman, and K. Keahey, "A scalable approach to deploying and managing appliances," in TeraGrid Conference (2007), Madison, WI, June 2007.

[11]. C. Peng, M. Kim, Z. Zhang, and H. Lei, "Vdn: Virtual machine image distribution network for cloud data centers," in INFOCOM, 2012 Proceedings. IEEE, 2012, pp. 181-189.

[12]. G. Kecskemeti, G. Terstyanszky, P. Kacsuk, and Z. Nemeth, "An approach for virtual appliance distribution for service deployment," 2011. 